\title{
Performance of Graphene Nanopowder-Polyvinyl Alcohol in Optical Pulse Generation at 1.5 Micron Region
}

\author{
Nabihah Hussin ${ }^{1}$, Mohd Rashidi Salim ${ }^{1}$, Asrul Izam Azmi ${ }^{1}$, Muhammad Yusof Mohd Noor ${ }^{1}$, \\ Ahmad Sharmi Abdullah ${ }^{1}$, Fauzan Ahmad², Mohd Haniff Ibrahim*1 \\ ${ }^{1}$ School of Electrical Engineering, Universiti Teknologi Malaysia, Johor, Malaysia, \\ ${ }^{2}$ Malaysia-Japan International Institute of Technology, Universiti Teknologi Malaysia, Kuala Lumpur, Malaysia.
}

Received September 22, 2021; accepted September 28, 2021; published September 30, 2021

\begin{abstract}
This paper explains the performance of a graphene nanopowder (GNP) based saturable absorber (SA) at the 1.5-micron region which is prepared by dissolution in polyvinyl alcohol (PVA) polymer. Two different GNP flake thicknesses (AO2-8 nm and AO4-60 $\mathrm{nm})$ are tested. By applying a solution casting method, three weight ratios of GNP to PVA (12.04, 8.03 and $3.11 \mathrm{wt} \%$ ) have been prepared and fabricated as a thin composite film. To characterize the SA performance, a $4 \mathrm{~mm}^{2}$ area of thin GNP-PVA film is embedded in a 14 meter long ring cavity with a 3 meter Erbium doped fiber (EDF) as a gain medium. Our characterization results show that the thin GNP-PVA film acts as a Q-switcher which produces stable laser pulses for 12.04 wt.\% with a maximum repetition rate of $39.22 \mathrm{kHz}$ and a shortest pulse width of $11.79 \mu \mathrm{s}$. Meanwhile, unstable Q-switched pulses of $8.03 \mathrm{wt} . \%$ and 3.11 wt.\% have been observed with recorded signal to noise ratio (SNR) of only $21 \mathrm{~dB}$ and $17 \mathrm{~dB}$, respectively. The threshold pumping power for Q-switched lasing to emerge is recorded as low as $30 \mathrm{~mW}$. Apparently, it shows that GNP concentration and flakes thickness in a fabricated SA composite plays a vital role in the performance of a generated Q-switch laser, particularly at the $1.5 \mu \mathrm{m}$ region.
\end{abstract}

Since its first theoretical exploration by Wallace in 1947, graphene has been well-researched in many related fields of engineering and science. The research of graphene in photonics covers broad areas, including the device and system levels such as light detection, transceivers, imaging system, solar cell development and pulse laser generation. Works on a graphene saturable absorber (SA) for pulse laser generation were first demonstrated in 2009 by Hasan et al. [1] and Bao et al. [2], in which ultra-short pulses were generated from a passively mode-locked fiber laser. Later, works on Q-switching pulse generation were published by Zheng et al. [3] and Popa et al. [4] in 2010 and 2011, respectively. These efforts were then continued by many researchers working on mode-locked pulses and Q-switch pulses generation.

Various methods have been proposed for graphenebased SA fabrication. These include chemical vapor deposition [2], mechanical exfoliation [5], ultrasonication [6], electrochemical exfoliation [7-8] and a dip coating method [9]. The selection of these methods is highly influenced by the realization cost, performance of fabricated SA and class of graphene. For instance, our previous work [7] adopted an electrochemical exfoliation technique to extract graphene from graphite electrodes.

*E-mail: mohdhaniff@utm.my
Jiang et al. [6] adopted a mild ultrasonication with sodium deoxycholate surfactant to produce graphene from graphite oxide reduction. In [5], Chang et al. demonstrated graphene mode-locked lasers by mechanical exfoliation of bulk graphite.

From our review, we noticed that there was a limited effort at realizing graphene nanopowder (GNP) as SA. Up to date, only few publications on graphene nanoplatelets which are a type of GNP have been demonstrated. This includes our previous work in [7] and work by Apandi, et al. [10] and Zalkepali et al. [11]. In these works, nanoplatelets dispersion was mixed with a host polymer at a fixed ratio to finally produce a graphene composite thin film. To the best of our knowledge, no work has been initiated to investigate the effect of GNP concentration in a host polymer for Q-switched pulse generation.

In this work, we will evaluate the performance of GNP as SA for different weight ratios of GNP to polyvinyl alcohol (PVA) polymer, which acts as the host material. For this purpose, two different GNP types (obtained from the Graphene Supermarket, NY, USA) were tested with a code of AO2 (8 nm flakes thickness) and AO4 $(60 \mathrm{~nm}$ flakes thickness). The PVA polymer in the form of solvent needs to be prepared for further mixing process with a graphene suspension. For this purpose, $120 \mathrm{ml} \mathrm{DI}$ water is used to dissolve $1 \mathrm{~g}$ of PVA by using a hot plate stirrer. The dispersion of $\mathrm{AO} 2$ and $\mathrm{AO} 4$ is achieved by 40 $\mathrm{mg}$ of nanopowder (AO2 and $\mathrm{AO} 4)$ addition into $40 \mathrm{ml}$ of $0.01 \mathrm{~g} / \mathrm{ml}$ of sodium dodecyl sulfate (SDS). To speed up the nanoparticles dispersion, sonication is carried out at $50 \mathrm{~W}$ for one hour. Centrifugation at $1000 \mathrm{rpm}$ for several minutes will be applied to remove big particles of undispersed $\mathrm{AO} 2$ and $\mathrm{AO} 4$, resulting in a dispersed suspension that remains stable for weeks. The dispersed GNP suspension is then mixed with a PVA solution to make $\mathrm{AO} 2$ and $\mathrm{AO} 4$ composites. To investigate the effect of volumetric ratio, three weight ratios of $\mathrm{AO} 2 / \mathrm{AO} 4$ to PVA are prepared (12.04, 8.03 and 3.11 wt. \%). Homogeneous composites can be obtained after one hour of ultrasonic processing. The suspensions are evaporated at room temperature for 48 hours, producing AO2-PVA and AO4-PVA film with a thickness of roughly $30 \mu \mathrm{m}$. The samples of fabricated film are shown in Fig. 1. 

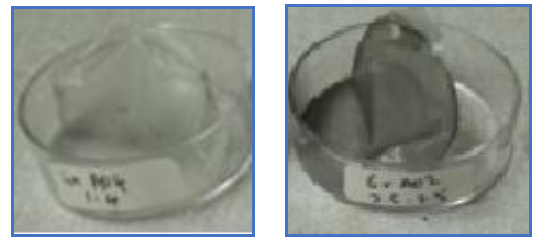

Fig. 1. Samples of fabricated GNP-based SA thin film.

Field Effect Scanning Microscopes (FESEM) Zeiss Crossbeam 340 are then used to characterize the physical embedment of graphene particles in the PVA host. The captured images in Fig. 2 indicate that $\mathrm{AO} 2$ and $\mathrm{AO} 4$ are randomly incorporated in the PVA composite film.
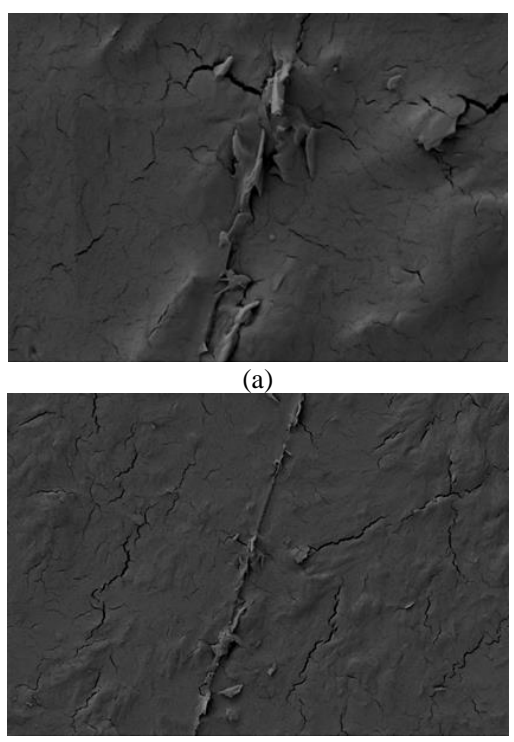

(b)

Fig. 2. FESEM images of (a) AO2-PVA and (b) AO4-PVA.

For SA characterization at the $1.5 \mu \mathrm{m}$ region, a configuration of EDF ring laser cavity is utilized as shown in Fig. 3. The gain medium used in this measurement is 3 meters long EDF. By using a 95/5 coupler, $5 \%$ of light will be tapped out for optical monitoring, characterization using oscilloscope (OSC) and optical spectrum analyzer (OSA). A radio frequency spectrum analyzer (RFSA) and an optical power meter (OPM) are also utilized in this setup. The EDFL spectrum, output pulse train, signal to noise ratio and average output power can be measured using this setup. As shown in Fig. 3, a small piece of AO2/AO4-PVA film (about $4 \mathrm{~mm}^{2}$ size) is integrated in the cavity with the aid of fiber ferrules and little addition of index matching gel.

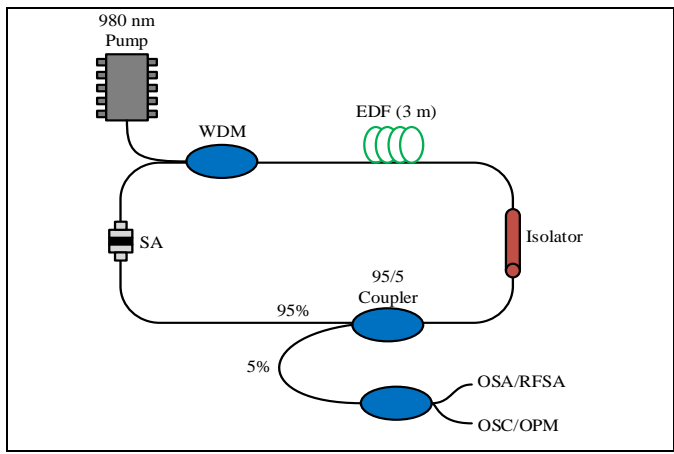

Fig. 3. Configuration of the EDFL cavity for $1.5 \mu \mathrm{m}$ characterization.

Figure 4 shows the optical spectrum of the Q-switched pulse for the AO4-PVA of 3.11 wt.\%. It shows that the central wavelength for a generated pulse is around 1568 $\mathrm{nm}$. Table 1 tabulates the central wavelength of the generated Q-switched pulse for AO2-PVA and AO4PVA. Note that for all GNP concentrations, the output spectrum of the Q-switched EDFL is broader and shifted to a shorter wavelength.

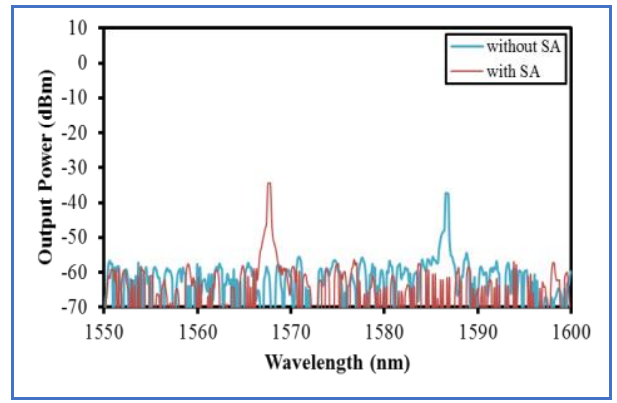

Fig. 4. OSA trace of AO4-PVA; for 1:4 ratio.

Oscilloscope traces for pulse train generated by $\mathrm{AO} 2-$ PVA at maximum pump power of $70 \mathrm{~mW}$ are shown in Fig. 5 for 12.04 wt.\%. From Table 1, AO4-PVA will create a shorter pulse width as compared to AO2-PVA, for all GNP concentrations. Figure 6 shows the repetition rate and pulse width as a function of pump power for 8.03 wt.\% of AO2-PVA. It demonstrates that Q-switched lasing starts at $35 \mathrm{~mW}$ up to a maximum pump power of $55 \mathrm{~mW}$. As a function of pump power, the repetition rate is measured to be proportionally varied while the pulse width shows the opposite pattern. Based on our measurement, AO4-PVA at 12.04 wt.\% exhibits the maximum repetition rate and shortest pulse width which stand at $39.22 \mathrm{kHz}$ and $11.79 \mu \mathrm{s}$, respectively. A detail comparison can be observed in Table 1, which indicates that the smallest recorded threshold pump power is 30 $\mathrm{mW}$ and the maximum can go up to $100 \mathrm{~mW}$. Calculated pulse energy and peak output power for maximum pump power are summarized in Table 1. It affirms that the pulse energy and peak power of AO4-PVA are higher compared to the AO2-PVA for all GNP concentrations. 


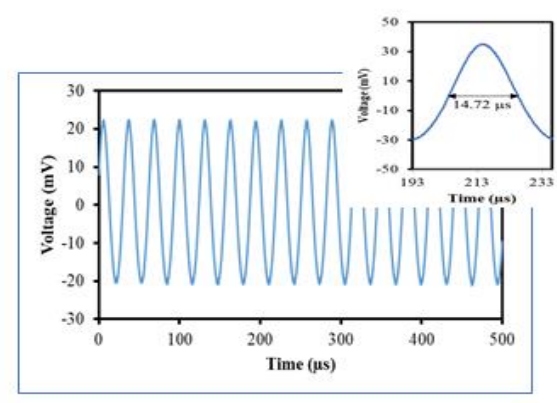

Fig. 5. Pulse train generated by AO2-PVA (1:1) at $70 \mathrm{~mW}$.

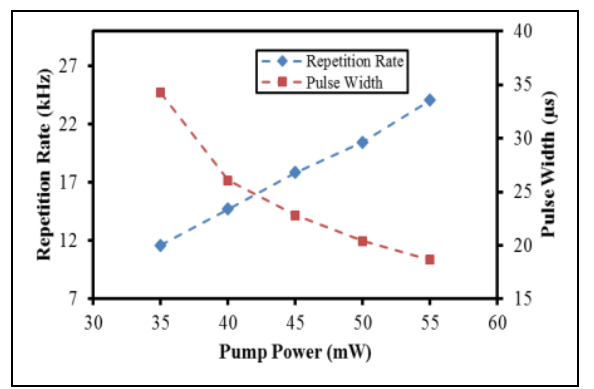

Fig.6. Repetition rate and pulse width for AO2-PVA (1:1.5).

By using the RFSA, radio frequency spectra for these SAs are recorded, which can verify the stability of generated pulse lasers. The plot is shown in Fig 7 for AO4-PVA at $12.04 \mathrm{wt}$ \%. Similarly, for AO2-PVA (12.04 wt.\%), $33 \mathrm{~dB}$ of SNR has been measured which vindicates the stability of generated pulses. However, the GNP concentrations of 8.03 and $3.11 \mathrm{wt} \%$ suggest the instability of generated pulses due to a smaller recorded value (less than $30 \mathrm{~dB}$ ).

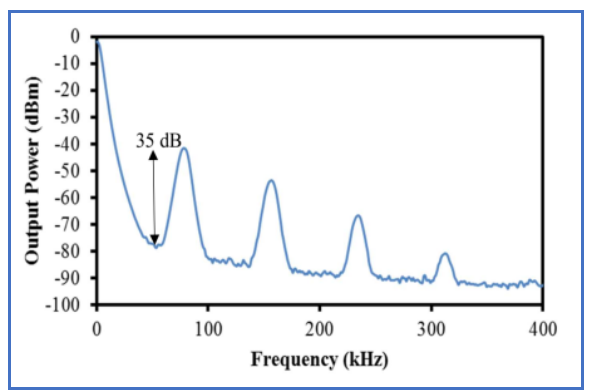

(b)

Fig. 7. RFSA measurement for AO4-PVA (1:1).

These findings assert the impact of GNP concentration in the PVA polymer composite SA film. In addition, the thickness of the flake vastly contributes to the SA performance. A comparative study on the effect of graphene concentration on a passive mode-locked fiber laser has been recorded in [12] being based on an optical deposition method. Nevertheless, we have successfully demonstrated for the first time, the effect of GNP concentration and flakes thickness on the performance of a generated Q-switched fiber laser at the $1.5 \mu \mathrm{m}$ region, based on GNP-PVA composite SA.

\begin{tabular}{|c|c|c|c|}
\hline \multirow{2}{*}{$\begin{array}{l}\text { Saturable Absorber } \\
\text { Weight ratio (GNP to PVA) }\end{array}$} & \multicolumn{3}{|c|}{ AO2-PVA } \\
\hline & 12.04 wt. $\%$ & 8.03 wt. $\%$ & 3.11 wt. $\%$ \\
\hline Range of pump power $(\mathrm{mW})$ & $30-70$ & $35-55$ & $35-60$ \\
\hline Threshold pump power(mW) & 30 & 35 & 35 \\
\hline Central wavelength $(\mathrm{nm})$ & 1566.0 & 1562.5 & 1570.4 \\
\hline Repetition rate $(\mathrm{kHz})$ & $10.79-31.85$ & $11.61-24.09$ & $9.62-20.24$ \\
\hline Shortest pulse width $(\mu \mathrm{s})$ & 14.72 & 18.66 & 23.1 \\
\hline Pulse energy (maximum) (nJ) & 3.33 & 4.73 & 12.5 \\
\hline Peak power (maximum) $(\mathrm{mW})$ & 0.21 & 0.24 & 0.51 \\
\hline SNR ( $\left(1^{\text {st }}\right.$ beat note $)(\mathrm{dB})$ & 33 & 21 & 17.2 \\
\hline Saturable Absorber & \multicolumn{3}{|c|}{ AO4-PVA } \\
\hline Weight ratio (GNP to PVA) & 12.04 wt. $\%$ & 8.03 wt. $\%$ & 3.11 wt. $\%$ \\
\hline Range of pump power $(\mathrm{mW})$ & $50-100$ & $30-100$ & $30-50$ \\
\hline Threshold pump power(mW) & 50 & 30 & 30 \\
\hline Central wavelength $(\mathrm{nm})$ & 1557.4 & 1567.2 & 1567.7 \\
\hline Repetition rate $(\mathrm{kHz})$ & $17.01-39.22$ & $7.65-33.20$ & $9.21-20.66$ \\
\hline Shortest pulse width $(\mu \mathrm{s})$ & 11.79 & 13.04 & 22.8 \\
\hline Pulse energy (maximum) (nJ) & 6.98 & 13.62 & 12.89 \\
\hline Peak power (maximum) $(\mathrm{mW})$ & 0.56 & 0.98 & 0.53 \\
\hline SNR ( $1^{\text {st }}$ beat note $)(\mathrm{dB})$ & 35 & 23 & 17 \\
\hline
\end{tabular}

Authors acknowledged the Ministry of Higher Education of Malaysia (R.J130000.7851.5F164) for the funding.

\section{References}

[1] T. Hasan, Z. Sun, F. Wang, F. Bonaccorso, P.H. Tan, A.G. Rozhin, A.C. Ferrari, Adv. Mater. 21, 3874 (2009).

[2] Q. Bao, H. Zhang, Y. Wang, Z. Ni, Y. Yan, Z.X. Shen, K.P. Loh, D.Y. Tang, Adv. Funct. Mater. 19, 3077 (2009).

[3] Z. Luo, M. Zhou, J. Weng, G. Huang, H. Xu, C. Ye, Z. Cai, Opt. Lett. 35, 3709 (2010)

[4] D. Popa, Z. Sun, T. Hasan, F. Torrisi, F. Wang, A.C. Ferrari, Appl. Phys. Lett. 98, 3106 (2011).

[5] Y.M. Chang, H. Kim, J.H. Lee, Y. Song, Appl. Phys. Lett. 97, 211102 (2010).

[6] M. Jiang, Z. Ren, Y. Zhang, B. Lu, R. Zhang, J. Guo, Y. Zhou, J. Bai, Mater. Sci. Forum 694, 700 (2011).

[7] N. Hussin, M.H. Ibrahim, F. Ahmad, H. Yahaya, S.W. Harun, Telkomnika 15, 814 (2017).

[8] F.C. Mat, M. Yasin, A.A. Latiff, S.W. Harun, Photonics Lett. Poland 9, 100 (2017).

[9] E.K. Ng, K.Y. Lau, H.K. Lee, N.M. Yusoff, A.R. Sarmani, M.F. Omar, M.A. Mahdi, Opt. Mater. Express 11, 59 (2021).

[10] N.H.M. Apandi, S.N.F. Zuikafly, N. Kasim, M.A. Mohamed, S.W. Harun, F. Ahmad, Bull. Electr. Eng. Inform. 8, 1358 (2019).

[11] N.U.H.H.B. Zalkepali, N.A. Awang, Y.R. Yuzaile, Z. Zakaria, A.A. Latif, F. Ahmad, J. Adv. Res. Dyn. Control Syst. 12, 602 (2020).

[12] X. Zhu and S. Chen, IEEE Photonics J. 11, 7105109 (2019). 